\title{
Computed tomography in predicting skin-epidural space distance for thoracic epidural catheterization
}

\author{
M.K. Arslantas, G.Tore Altun, A. Kararmaz \\ Marmara University, School of Medicine, Department of Anaesthesiology - Istanbul (Turkey)
}

\section{Background and Goal of Study:}

Thoracic epidural analgesia is a choice of analgesic method in thoracic surgery. Thoracic epidural catheter insertion is a technically challenging procedure and has serious neurologic complications. To know skin-epidural distance before the procedure may be helpful to identify epidural space with Tuohy needle. We aimed to compare the distance of skin to epidural space measured by CT scan and Tuohy needle.

\section{Materials and Methods:}

This prospective study enrolled 25 patients who underwent elective major thoracic surgery and required high thoracic epidural catheterization for postoperative pain management. By using INFINITT PACS software, the maximum and minimum distance of skin to epidural space were measured from the sagittal slice of the thoracic CT images (Figure 1). With patients placed in sitting position epidural catheterization was performed from the midline of the T4-T5 or T5-T6 intervertebral space. The actual distance was measured from the needle when the epidural space was entered.
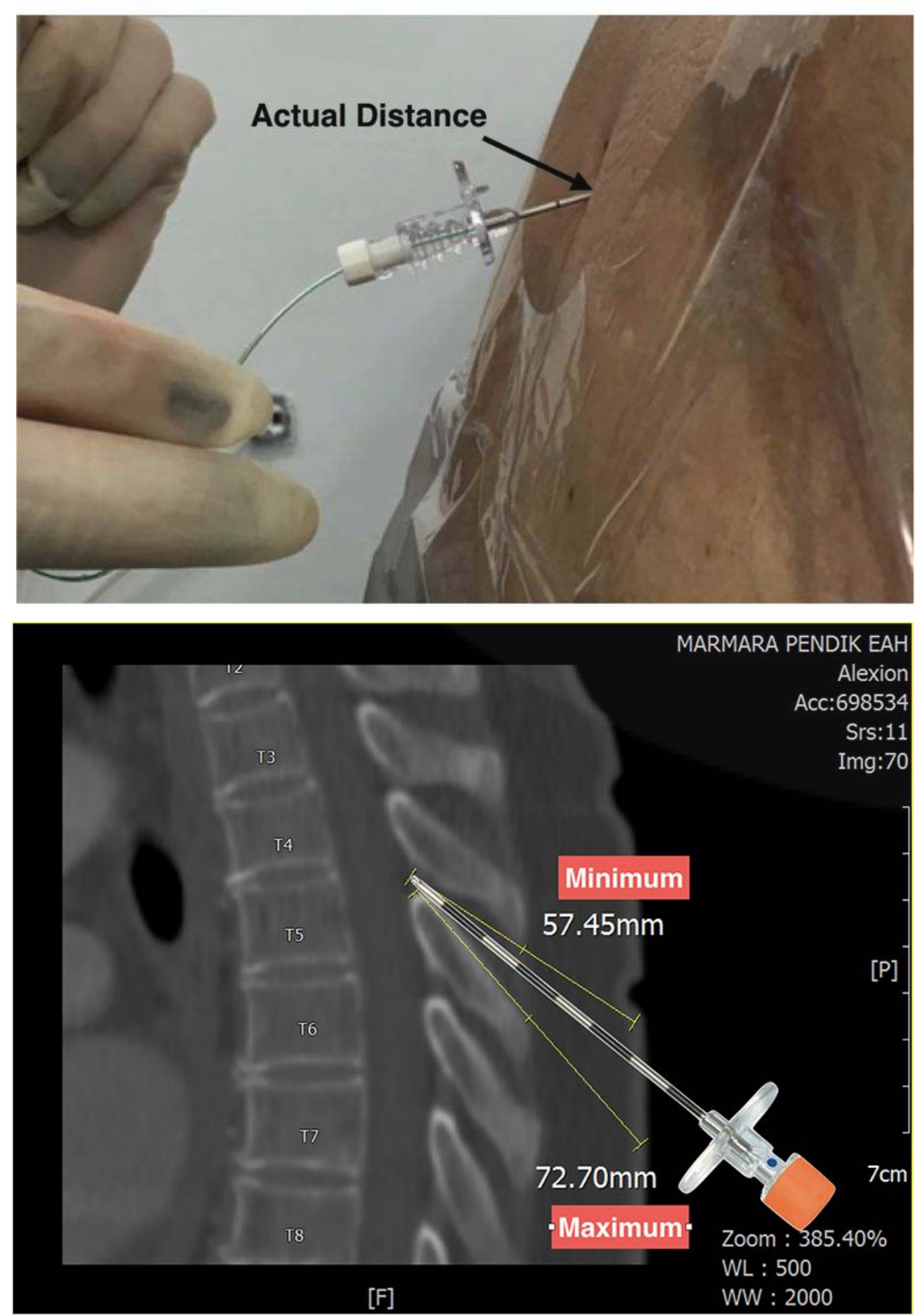
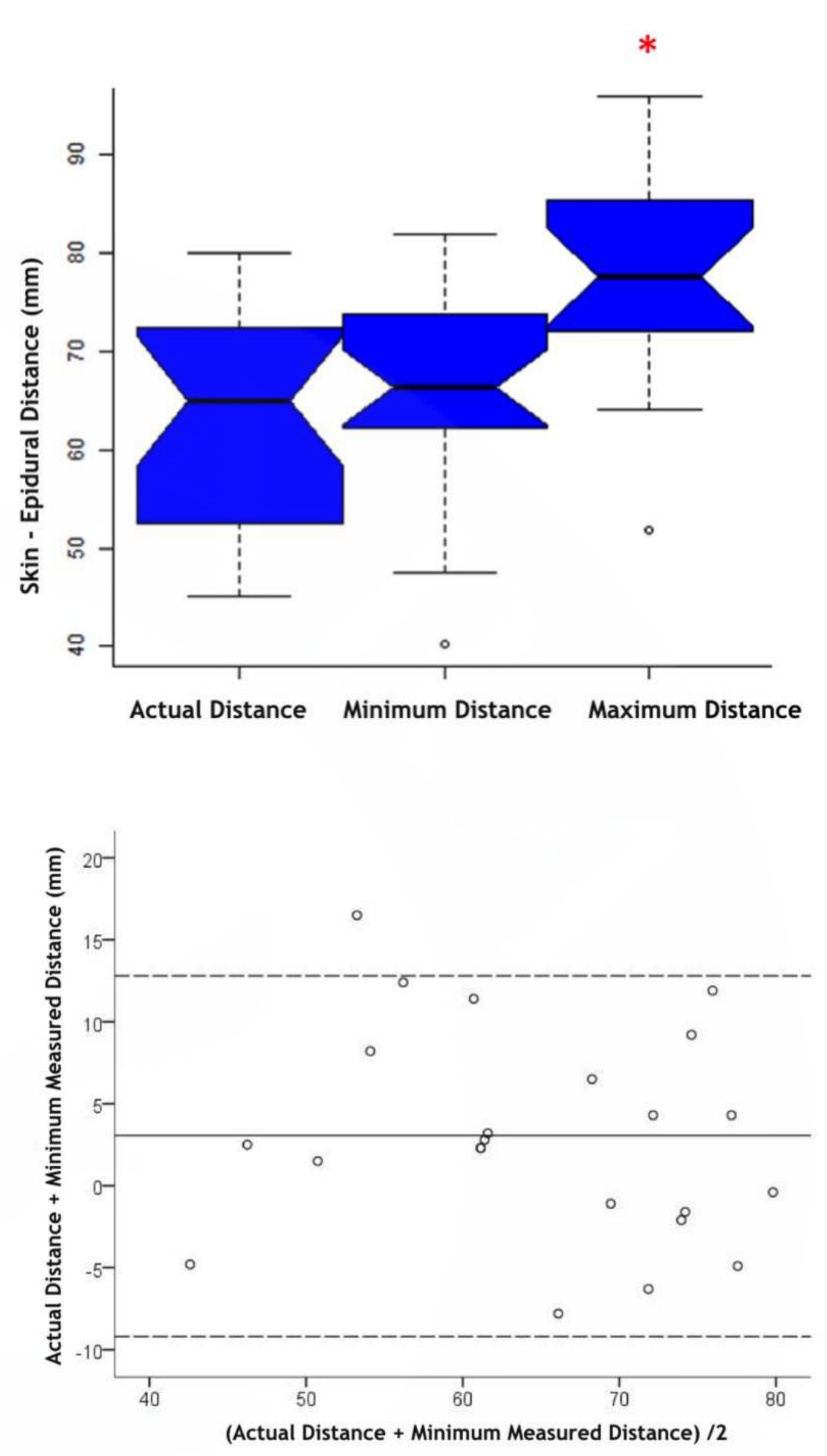

Results and Discussion:

There was no statistically significant difference between minimum skin-epidural space distance measured from the CT images and actual measurements. However, the maximum distance measured from the CT images was significantly different from actual measurements $(p<0.0001)$. Bland-Altman analysis for actual distance and minimum skin-epidural space distance obtained from $\mathrm{CT}$ images revealed that these two methods could be used interchangeably.

\section{Conclusion(s):}

Preoperative thorax CT images could be used to predict skin to epidural space distance for high thoracic epidural catheterization done from midline approach. 